\title{
Density of warm ionized gas near the Galactic center: low radio frequency observations
}

\author{
Subhashis Roy \\ NCRA-TIFR, Pune University Campus, \\ Pune-411007, India \\ email: roy@ncra.tifr.res.in
}

\begin{abstract}
We have detected 62 compact likely extragalactic sources in the Galactic center (GC) region at 0.154 and $0.255 \mathrm{GHz}$ with the Giant Metrewave Radio Telescope (GMRT). Their scattering sizes go down linearly with angular distance from the GC up to about $1^{\circ}$. These are more than 10 times lower than the proposed Hyperstrong scattering model within $0.5^{\circ}$ from the GC. We also detect 7 out of $10 \mathrm{EG}$ sources expected in the region from existing catalogs. Ionized interfaces of dense molecular clouds to the ambient medium are likely responsible for strong scattering. However, dense GC clouds traced by CS $J=(1-0)$ emission are found to have a narrow distribution of $\sim 0.2^{\circ}$ across the Galactic plane. Lack of strong scattering towards EG sources within $\sim 0.5^{\circ}$ from GC with $|b| \gtrsim 0.2^{\circ}$ could explain our results.
\end{abstract}

Keywords. Galaxy: center - ISM: clouds - radio continuum: ISM - H II regions

\section{Introduction}

The central few hundred parsec region of the Galaxy is characterized by a dense and turbulent interstellar medium, and the central compact source Sgr A* is known to be scatter broadened to about $1^{\prime \prime}$ near $1 \mathrm{GHz}$. Lazio \& Cordes (1998) for the first time predicted the electron density in the central $\sim 100 \mathrm{pc}$ region. They predict the electron density in the region to be about $10 \mathrm{~cm}^{-3}$. This model predicts scattering sizes of extragalactic sources seen through it as $\sim 100^{\prime \prime}$ at $1 \mathrm{GHz}$. However, the scattering sizes of EG sources are inversely proportional to the distance of the screen from the Galactic center (GC). In the last one decade, some of the constraints used in the model have changed. For example, emission from $\mathrm{Sgr} \mathrm{A}^{*}$ is shown not to undergo free-free absorption due to Sgr A West below $950 \mathrm{MHz}$, but has been observed at much lower frequencies (Nord et al. 2004; Roy \& Pramesh Rao 2004).

The scattering size is roughly proportional to the square of the observing wavelength. We have carried out Giant Metrewave radio telescope (GMRT) observations at 0.255 and $0.154 \mathrm{GHz}$ of the central $2^{\circ}$ region of the $\mathrm{GC}$ to detect a large number of background EG sources, and measure their angular sizes at more than one wavelength.

\section{Results and discussions}

\subsection{Scattering diameters of compact sources and the GCWIM}

Observing details, sources properties at different frequencies, scattering diameters at $\lambda=$ $1 \mathrm{~m}$ and detailed results have been presented in Roy (2013). As plotted in Figure 1 of Roy (2013), scattering sizes go down linearly from the GC as a function of angular distance up to about $1.0^{\circ}$. It also shows the scattering size of sources at 
$\lambda=1$ meter at the GC is only about $30^{\prime \prime}$, and falls down to about $4^{\prime \prime}$ about a degree away from it. The ratio of apparent scattering diameter of an EG source to a GC source is given by $\frac{\theta_{s(E G)}}{\theta_{s(G C)}}=\frac{D}{d}$ (Lazio \& Cordes 1998), where D is GC to observer distance and ' $d$ ' is screen to GC distance. If the electron density fluctuation parameter does not change significantly in the region, it would indicate $\int n_{e}^{2} . d l$ to also fall down in similar fashion. Since the peak of scattering is coincident with the GC, this indicates that indeed there is a GC component of scattering seen within $\sim 1^{\circ}$ from the GC. At the distance of the GC, its linear size is $\sim 100 \mathrm{pc}$. However, the scattering size produced by this gas at $1 \mathrm{GHz}$ is only $\sim 3^{\prime \prime}$, more than an order of magnitude smaller than what is expected from the Hyperstrong scattering screen. To better constrain the Hyperstrong scattering model, we have also checked for the number of EG sources seen through the $359.5^{\circ}<l<0.5^{\circ}$ and $-0.5^{\circ}<b<0.5^{\circ}$ region of the Galaxy. Seven likely EG sources have been identified out of 10 expected (Roy 2013). The results could be explained if the electron density towards the EG sources is almost an order of magnitude smaller than predicted from the above model, or the scattering medium is several kpc away from the GC (Bower et al. 2014).

\subsection{Free-free absorption towards extended non-thermal GC sources}

Free-free absorption depth $(\tau)$ towards the well known extended sources in the GC region at $0.154 \mathrm{GHz}$ has been tabulated in Roy (2013). Higher values (>0.5) of $\tau$ were only obtained for sources within $0.6^{\circ}$ from the GC. $\tau$ vary widely towards different extended sources. The angular scale of absorption is $\sim 10^{\prime}$. This corresponds to a length scale of $\sim 25 \mathrm{pc}$ at the distance of the GC, and indicates that absorption within $0.6^{\circ}$ from the $\mathrm{GC}$ is caused by ionized clouds whose emission measure is $\sim 10^{4} \mathrm{~cm}^{-6} \mathrm{pc}$. Their length scale implies electron densities in the clouds to be $\sim 10 \mathrm{~cm}^{-3}$.

\subsection{GC molecular cloud distribution and the scattering medium}

The surfaces of molecular clouds in the GC region are mostly ionized by external radiation and will cause large scattering due to high electron density in the region (Yusef-Zadeh et al. 1994; Lazio \& Cordes 1998). The GC molecular clouds are characterized by their high density and therefore could be identified by CS $J=(1-0)$ emission. This emission was mapped by Tsuboi et al. (1999), and we find from the moment zero map (their Figure 2(a)) that the clouds have a narrow scale height near the GC, and are typically seen within $-0.2^{\circ}<b<0.1^{\circ}$. Angular distribution of most of the EG sources seen through the so called Hyperstrong scattering region are random in $b$, and typically $\sim 7$ out of 10 sources will not be seen through the dense molecular clouds. Asymmetric distribution of the screen around the GC could explain why most of the EG sources seen through the region are not scatter broadened at $1.4 \mathrm{GHz}$. This also explains the large $\tau$ towards the extended GC non-thermal sources, most of which are seen close to the Galactic plane.

\section{References}

Bower, G. C., Deller, A., \& Brunthaler A. et al. 2014, ApJ 780L, 2

Lazio, T. J. W. \& Cordes, J. M. 1998, ApJ 505, 715

Nord, M. E., Lazio, T. J. W., Kassim, N. E., et al. 2004, AJ 128, 1646

Roy, S. \& Pramesh Rao, A. 2004, MNRAS 349, L25

Roy, S. 2013, ApJ 773, 67

Tsuboi, M., Handa, T., \& Ukita, N. 1999, ApJS 120, 1

Yusef-Zadeh, F., Cotton, W., Wardle, M., Melia, F., \& Roberts, D. A. 1994, ApJ Lett. 434, L63 